\title{
Lateralization of Circadian Pacemaker Output: Activation of Left- and Right-Sided Luteinizing Hormone-Releasing Hormone Neurons Involves a Neural Rather Than a Humoral Pathway
}

\author{
Horacio 0. de la Iglesia, Jennifer Meyer, and William J. Schwartz \\ Department of Neurology, University of Massachusetts Medical School, Worcester, Massachusetts 01655
}

\begin{abstract}
Locomotor activity and luteinizing hormone (LH) secretion in golden hamsters share a common circadian pacemaker in the suprachiasmatic nucleus (SCN), but the rhythms do not seem to share a common output pathway from the SCN. Locomotion is believed to be driven by humoral factor(s), whereas LH secretion may depend on specific ipsilateral neural efferents from the SCN to LH releasing hormone (LHRH)-containing neurons in the preoptic area. In this paper we provide the first functional evidence for such efferents in neurologically intact hamsters by exploiting a phenomenon known as "splitting" in constant light, in which circa-12 hr (approximately 12 hr) locomotor activity bouts reflect an antiphase oscillation of the left and right sides of the bilaterally paired SCN. In ovariectomized, estrogen-treated (OVX $\left.+\mathrm{E}_{2}\right)$ female hamsters, splitting is also known to include circa-12 hr LH secretory surges. Here we show that behaviorally "split" $\mathrm{OVX}+\mathrm{E}_{2}$ females exhibit a marked left-right asymmetry in immunoreactive c-Fos expression in both SCN and activated LHRH neurons, with the percentage of $\mathrm{LHRH}^{+} / \mathrm{c}$-Fos ${ }^{+}$double-labeled cells approximately fivefold higher on the side corresponding to the side of the SCN with higher c-Fos immunoreactivity. Our results suggest that splitting involves alternating left- and right-sided stimulation of LHRH neurons; under such circumstances, the functional activity of the neuroendocrine hypothalamus mirrors intrinsic side-to-side differences in SCN gene expression. The circadian regulation of reproductive activity depends on lateralized, point-to-point axonal projections rather than on diffusible factors.
\end{abstract}

Key words: gonadotropin; hypothalamus; luteinizing hormone; preoptic; suprachiasmatic; fos

\section{Introduction}

The master circadian pacemaker in mammals, located in the suprachiasmatic nucleus (SCN) of the hypothalamus, governs a diverse array of behavioral, physiological, and hormonal rhythms, all of which express different phases and waveforms (for review, see Herzog and Schwartz, 2002). How the SCN organizes this temporal program is not well understood and appears to be complex. In golden hamsters, circadian regulation of both locomotor (wheel-running) activity and luteinizing hormone (LH) secretion is abolished by SCN lesions (Turek and Van Cauter, 1988), and both rhythms are altered in parallel in the shortperiod phenotype (tau) caused by mutation of the casein kinase $1 \epsilon$ gene (Lucas et al., 1999). Despite sharing a common pacemaker in the SCN, however, the rhythms do not seem to share a common output pathway from the SCN. Circadian wheel running is believed to be driven by humoral factor(s) released by SCN cells. The rhythm persists after surgical isolation of the SCN as a hypothalamic island in situ (Hakim et al., 1991), and it is

Received April 28, 2003; revised June 12, 2003; accepted June 25, 2003.

This research was supported by the National Institute of Neurological Disorders and Stroke.

Correspondence should be addressed to Horacio 0 . de la Iglesia, Department of Biology, University of Washington, Box 351800, Seattle, WA 98195-1800. E-mail: horaciod@u.washington.edu.

Copyright $\odot 2003$ Society for Neuroscience $\quad$ 0270-6474/03/237412-03\$15.00/0 restored in arrhythmic, SCN-lesioned animals by transplantation of SCN tissue encased in semipermeable capsules (Silver et al., 1996). On the other hand, circadian LH secretion and reproductive cycles are abolished by discrete knife cuts dorsocaudal to the SCN in rats (Watts et al., 1989), and they are not restored by transplants in hamsters (Meyer-Bernstein et al., 1999). These data have raised the possibility that specific neural efferents from the SCN carry the output signal for this function, presumably via a direct, predominantly ipsilateral projection to $\mathrm{LH}$ releasing hormone (LHRH)-containing neurons in the preoptic area (de la Iglesia et al., 1995; van der Beek et al., 1997), but interpretation of arrhythmicity in these experiments is compromised by nonspecific surgical effects. In this report, we exploit an unusual property of the circadian system to investigate LHRH neuronal activation in neurologically intact animals and to implicate a neural rather than humoral mechanism for the control of this neuroendocrine output by the SCN.

Hamsters maintained in constant light (LL) can exhibit "splitting" of their locomotor activity rhythm, in which the single daily bout of activity dissociates into two components stably coupled $180^{\circ}(\sim 12 \mathrm{hr}$ ) apart. In ovariectomized, estrogen-treated (OVX $+\mathrm{E}_{2}$ ) female hamsters, splitting also includes circa-12 hr (approximately $12 \mathrm{hr}$ ) LH secretory surges (Swann and Turek, 1985); behaviorally "unsplit" OVX $+\mathrm{E}_{2}$ females exhibit only a 
single daily surge in LL. The splitting phenomenon is believed to represent the activity of two circadian oscillators cycling oppositely in antiphase, and we have shown recently that these two oscillators correspond to the left and right sides of the bilaterally paired SCN (de la Iglesia et al., 2000). We reasoned that if the ipsilateral innervation of LHRH neurons by the SCN mediates circadian gating of the LH surge, then each surge in a "split" female might reflect the activation of either left- or right-sided LHRH neurons. We tested this prediction, identifying activated LHRH neurons by their expression of immunoreactive c-Fos $1 \mathrm{hr}$ before locomotor activity onset (Doan and Urbanski, 1994), just after the start of the expected LH surge.

\section{Materials and Methods}

Female golden hamsters (Charles River, Kingston, NY), $21 \mathrm{~d}$ old at time of delivery, were used in accordance with the regulations of the Univer-

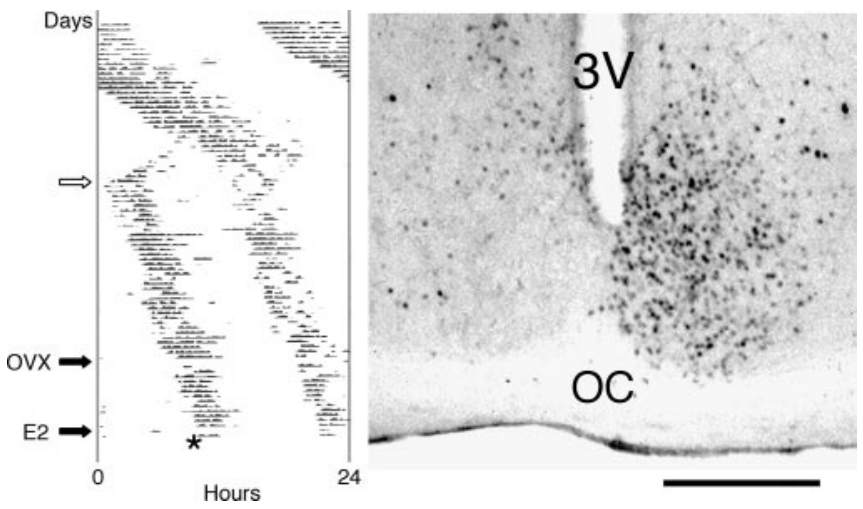

Figure 1. Behavioral splitting and immunoreactive c-Fos expression in the SCN. Left, Wheelrunning activity of a representative female hamster maintained in $\mathrm{L} L$, with the number of wheel revolutions over the course of each $24 \mathrm{hr}$ period charted horizontally from left to right and succeeding days stacked vertically from top to bottom. At the white arrow, the animal's single daily bout of activity dissociated into two split components stably coupled $\sim 12 \mathrm{hr}$ apart ( $\sim 30 \%$ of females exposed to LL exhibited splitting within $90 \mathrm{~d}$ ). The animal was then ovariectomized (OVX), subcutaneously implanted with an estradiol benzoate capsule $14 \mathrm{~d}$ later (E2), and killed after $2 \mathrm{~d}$ (asterisk); $20 \%$ of the females, whether split or nonsplit, had disrupted wheel-running rhythms after OVX and were discarded from the study. Right, Coronal brain section through the $\mathrm{SCN}$ of a behaviorally split female hamster, killed $1 \mathrm{hr}$ before the onset of a split activity bout and processed for c-Fos immunohistochemistry. $3 \mathrm{~V}$, Third ventricle; $\mathrm{OC}$, optic chiasm. Scale bar, $500 \mu \mathrm{m}$.

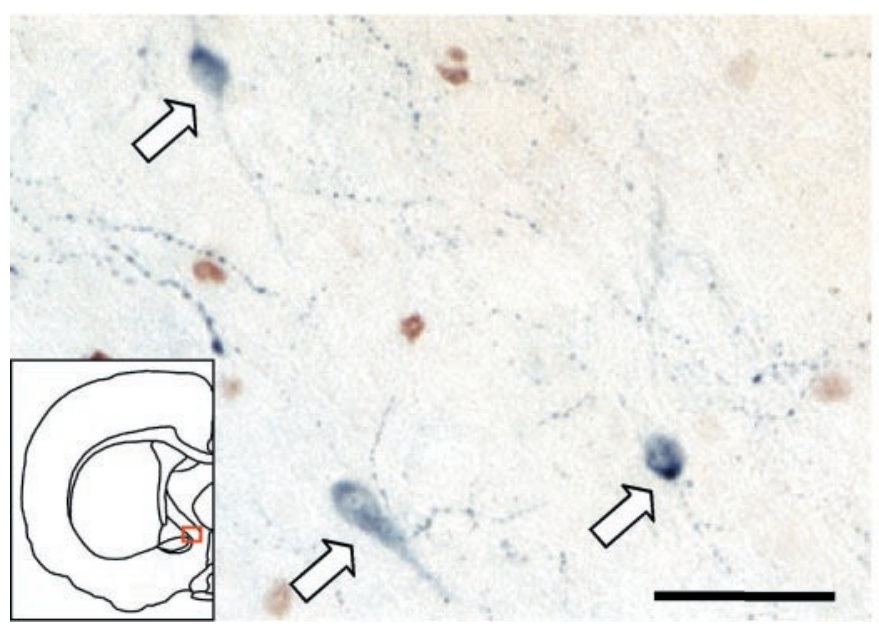

sity of Massachusetts Institutional Animal Care and Use Committee. They were housed individually in LL (300-400 lux), and wheel-running activity was recorded continuously to monitor for split activity rhythms (see Fig. 1). Behaviorally split and nonsplit control females were ovariectomized under ketamine-xylazine anesthesia, returned to their cages for an additional $14 \mathrm{~d}$ in LL, and then each was anesthetized and implanted subcutaneously with two 1-cm-long SILASTIC capsules filled with powdered estradiol benzoate (EB). Two days after EB implantation, behaviorally split animals were killed $1 \mathrm{hr}$ before the onset of either of their two split activity bouts, and nonsplit animals were killed either $1 \mathrm{hr}(1 \mathrm{hr}$ controls) or $13 \mathrm{hr}$ (13 hr controls) before the onset of their single daily activity bout. After anesthetic overdose, animals were perfused with PBS followed by $4 \%$ paraformaldehyde in $0.1 \mathrm{M}$ phosphate buffer, brains were removed and postfixed overnight at $4^{\circ} \mathrm{C}$, and $50 \mu \mathrm{m}$ coronal sections were cut from the lateral septum and diagonal band through the preoptic area to the SCN. Double-label immunohistochemistry was performed by simultaneous incubation with rabbit anti-c-Fos (1:10,000; SC52, Santa Cruz Biotechnology, Santa Cruz, CA) and mouse anti-LHRH (1:1000; $\mathrm{HU} 4 \mathrm{H}$, generous gift of Dr. H. F. Urbanski, Oregon Health and Science University, Portland, OR) for $72 \mathrm{hr}$ at $4^{\circ} \mathrm{C}$, followed by treatment with biotinylated goat anti-rabbit-diaminobenzidine and goat antimouse-SG kit (Vector Laboratories, Burlingame, CA), respectively. Alternate sections were prepared for immunofluorescence and confocal microscopy using Alexa 594 goat anti-rabbit and Alexa 488 goat antimouse secondary antibodies (1:200; Molecular Probes, Eugene, OR). Excitation wavelengths for Alexa 594 and 488 were 568 and $488 \mathrm{~nm}$, respectively. For each animal, the total number of $\mathrm{LHRH}^{+}$single-labeled and $\mathrm{LHRH}^{+} / \mathrm{c}-\mathrm{Fos}^{+}$double-labeled cells was counted on a set of sections spanning the rostral lateral septum to the caudal preoptic area $[15.1 \pm 0.5$ sections (mean \pm SE) counted for each animal], and the percentage of double-labeled cells was calculated separately for the left and right sides of the brain. Also counted was the number of c-Fos ${ }^{+}$cells in the section of the SCN that represented its largest mediolateral and ventrodorsal extent.

\section{Results}

As we reported previously for male hamsters (de la Iglesia et al., 2000), behaviorally split OVX $+E_{2}$ female hamsters exhibited a dramatic left-right asymmetry in immunoreactive c-Fos expression in the SCN (Fig. 1), with $323 \pm 18$ labeled cells on the "high" side (whether left or right) and $59 \pm 9$ labeled cells on the "low" side $(n=10)$. In the septal-preoptic area of these animals, $\mathrm{LHRH}^{+}$single-labeled and $\mathrm{LHRH}^{+} / \mathrm{c}$-Fos ${ }^{+}$double-labeled cells were present on both sides of the brain, but the percentage of double-labeled cells was approximately fivefold higher on the

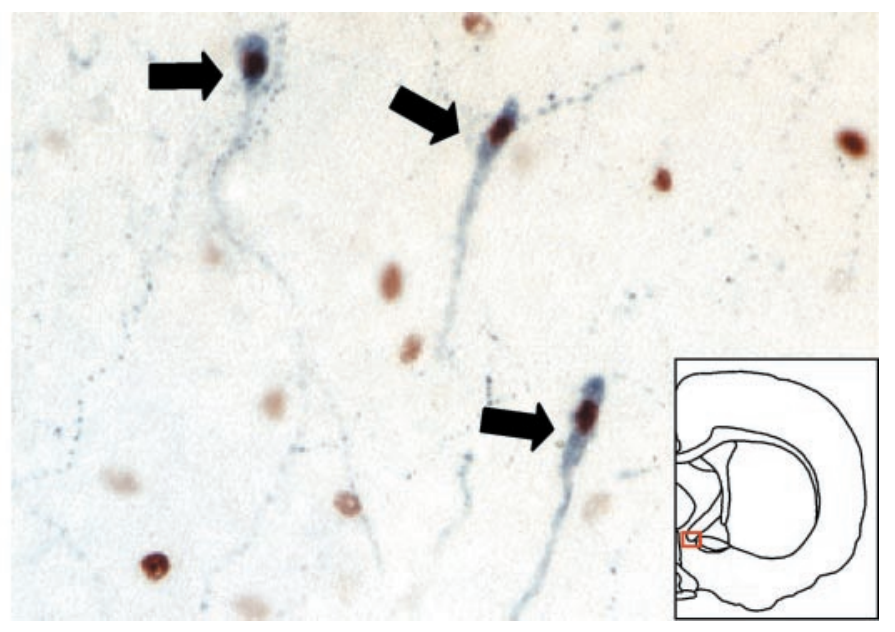

Figure 2. Asymmetric activation of LHRH-containing neurons during splitting. LHRH ${ }^{+}$(blue) single-labeled cells (white arrows, left) and LHRH ${ }^{+}$(blue)/c-Fos ${ }^{+}$(brown) double-labeled cells (black arrows, right) from the left and right sides (red squares in insets) of the brain of the hamster whose $\mathrm{SCN}$ is shown in Figure 1. Similar results were observed using confocal microscopy (0.5- $\mu \mathrm{m}$-thick optical sections) and immunofluorescence (data not shown). Scale bar, $50 \mu \mathrm{m}$. 
side corresponding to the side of the SCN with higher c-Fos immunoreactivity (Fig. 2). In behaviorally split OVX $+\mathrm{E}_{2}$ females, $21.2 \pm 4.0 \%$ of $\mathrm{LHRH}^{+}$cells were also c-Fos ${ }^{+}$on the side ipsilateral to high SCN c-Fos expression $\left(64 \pm 7\right.$ total $\mathrm{LHRH}^{+}$cells per side per animal), but only $4.4 \pm 1.2 \%$ were double labeled on the side contralateral to high SCN c-Fos expression ( $64 \pm 6$ total $\mathrm{LHRH}^{+}$cells per side per animal). In nonsplit OVX $+\mathrm{E}_{2} 1 \mathrm{hr}$ controls $(n=7)$, there was no such side-to-side asymmetry $(12.3 \pm 3.4$ and $11.4 \pm 4.4 \%$ double-labeled cells, with $69 \pm 10$ and $68 \pm 10$ total $\mathrm{LHRH}^{+}$cells per side per animal; the high SCN side in nonsplit animals was defined as the side with marginally higher cell counts). The side-to-side difference in the percentage of double-labeled cells in the behaviorally split group, but not in the nonsplit group, was statistically significant ( $p=0.002$; twotailed $t$ test). The absolute number of double-labeled cells per animal (regardless of side) was not significantly different between the split and nonsplit groups (17.1 \pm 5.4 and $16.9 \pm 3.8)$, raising the possibility that this might represent the minimum number of activated LHRH cells necessary for an LH surge in OVX $+\mathrm{E}_{2}$ animals. As expected, no double-labeled cells were observed in nonsplit 13-hr controls $(n=2)$.

Of note, one of the seven nonsplit $1 \mathrm{hr}$ controls included in the above analysis exhibited a marked left-right asymmetry in the percentage of $\mathrm{LHRH}^{+} / \mathrm{c}-\mathrm{Fos}^{+}$double-labeled cells (24.1 vs $2.0 \%)$, along with a correspondingly asymmetrical pattern of SCN c-Fos expression (268 vs 93 labeled cells, compared with $241 \pm 29$ vs $200 \pm 30$ labeled cells in the remaining $1 \mathrm{hr}$ controls). Although this was the only such animal in the control group, this case hints that a time delay might exist between the onset of splitting within the SCN oscillatory apparatus and its subsequent reflection as split bouts of wheel-running activity.

\section{Discussion}

Asymmetric activation of LHRH neurons in behaviorally split hamsters provides a unique functional demonstration of the ipsilateral innervation of these neurons by each side of the SCN, heretofore identified solely by lesion, histochemical, and tracttracing methods (de la Iglesia et al., 1995; van der Beek et al., 1997). During splitting, we know that the SCN is reorganized as two oppositely phased, left- and right-sided circadian pacemakers, and the present data suggest that such a split SCN also activates bilateral LHRH neurons in antiphase. Alternating left- and right-sided stimulation of these neurons likely accounts for the generation of the circa-12 hr LH secretory surge in behaviorally split females. Thus, at least under these circumstances, the functional activity of neuroendocrine hypothalamus mirrors an intrinsic side-to-side difference in SCN gene expression.

Our results point to an organization of SCN output pathways that is multimodal. Although locomotor activity may be regulated by diffusible factors released by SCN cells (Kramer et al., 2001; Cheng et al., 2002), reproductive activity depends instead on lateralized, point-to-point axonal projections to specific regional targets. The multiplicity of SCN output mechanisms could provide a substrate for independent adjustment of the temporal sequencing of disparate clock-controlled events.

\section{References}

Cheng MY, Bullock CM, Li C, Lee AG, Bermak JC, Belluzzi J, Weaver DR, Leslie FM, Zhou Q-Y (2002) Prokineticin 2 transmits the behavioural circadian rhythm of the suprachiasmatic nucleus. Nature 417:405-410.

de la Iglesia HO, Blaustein JD, Bittman EL (1995) The suprachiasmatic area in the female hamster projects to neurons containing estrogen receptors and GnRH. NeuroReport 6:1715-1722.

de la Iglesia HO, Meyer J, Carpino Jr A, Schwartz WJ (2000) Antiphase oscillation of the left and right suprachiasmatic nuclei. Science 290:799-801.

Doan A, Urbanski HF (1994) Diurnal expression of Fos in luteinizing hormone-releasing hormone neurons of Syrian hamsters. Biol Reprod 50:301-308.

Hakim H, DeBernardo AP, Silver R (1991) Circadian locomotor rhythms, but not photoperiodic responses, survive surgical isolation of the SCN in hamsters. J Biol Rhythms 6:97-113.

Herzog ED, Schwartz WJ (2002) A neural clockwork for encoding circadian time. J Appl Physiol 92:401-408.

Kramer A, Yang F-C, Snodgrass P, Li X, Scammell TE, Davis FC, Weitz CJ (2001) Regulation of daily locomotor activity and sleep by hypothalamic EGF receptor signaling. Science 294:2511-2515.

Lucas RJ, Stirland JA, Darrow JM, Menaker M, Loudon ASI (1999) Free running circadian rhythms of melatonin, luteinizing hormone, and cortisol in Syrian hamsters bearing the circadian tau mutation. Endocrinology 140:758-764.

Meyer-Bernstein EL, Jetton AE, Matsumoto S, Markuns JF, Lehman MN, Bittman EL (1999) Effects of suprachiasmatic transplants on circadian rhythms of neuroendocrine function in golden hamsters. Endocrinology 140:207-218.

Silver R, LeSauter J, Tresco PA, Lehman MN (1996) A diffusible coupling signal from the transplanted suprachiasmatic nucleus controlling circadian locomotor rhythms. Nature 382:810-813.

Swann JM, Turek FW (1985) Multiple circadian oscillators regulate the timing of behavioral and endocrine rhythms in female golden hamsters. Science 228:898-900.

Turek FW, Van Cauter E (1988) Rhythms in reproduction. In: The physiology of reproduction (Knobil, E, Neill J, eds), pp 1789-1831. New York: Raven.

van der Beek EM, Horvath TL, Wiegant VM, van den Hurk R, Buijs RM (1997) Evidence for a direct neuronal pathway from the suprachiasmatic nucleus to the gonadotropin-releasing hormone system: combined tracing and light and electron microscopic immunocytochemical studies. J Comp Neurol 384:569-579.

Watts AG, Sheward WJ, Whale D, Fink G (1989) The effect of knife cuts in the subparaventricular zone of the female rat hypothalamus on oestrogen-induced diurnal surges of plasma prolactin and LH and circadian wheel-running activity. J Endocrinol 122:593-604. 